\title{
A DIVERGENT, TWO-PARAMETER, BOUNDED MARTINGALE
}

\author{
LESTER E. DUBINS ${ }^{1}$ AND JIM PITMAN
}

\begin{abstract}
An example is given of a divergent, uniformly bounded martingale $X=\left\{X_{t}: t \in T\right\}$ where the index $t$ ranges over the set $T$ of pairs of positive integers with the usual coordinatewise ordering.
\end{abstract}

This note offers an example of a divergent, uniformly bounded, two-parameter martingale which supplements the infinite-parameter example of Dieudonné [3]. Also offered here is a divergent, uniformly bounded, two-parameter, reversed martingale simpler than the one mentioned in [4].

For $m$ a positive integer and $0<\varepsilon<1$ an $(m, \varepsilon)$-daisy is a partition $\Pi$ of the universal event consisting of $m+1$ events $C, B_{1}, \ldots, B_{m}$ where $C$, the center of the daisy, has probability $\varepsilon$, and the $B_{i}$ have equal probability $(1-\varepsilon) / m$. Let $\Pi_{i}$ be the two-element partition consisting of $C \cup B_{i}$ and its complement. Plainly, the value of $P\left(C \mid \Pi_{i}\right)$ on $C \cup B_{i}$ is $(1+(1-\varepsilon) / m \varepsilon)^{-1}$, which is now abbreviated to $c(\varepsilon, m)$. Consequently, $\sup _{1<i<m} P\left(C \mid \Pi_{i}\right)=c(\varepsilon, m)$ everywhere. Indeed, a simple calculation shows that, for any pair $s$ of positive integers $a, b$,

$$
\sup _{a<i<m-b} P\left(C \mid \Pi_{i}\right)=c(\varepsilon, m)
$$

with probability greater than $1-|s| / m$, where $|s|$ is $a+b$.

Let $T$ be the set of all ordered couples of positive integers endowed with the coordinatewise ordering, that is, $s \leqslant t$ if each coordinate of $t-s$ is nonnegative.

An array of partitions $\Pi_{t}, t \in T$, is based on the $(m, \varepsilon)$-daisy $\Pi$ if $|t|=m$ and $t=(i, j)$ implies that $\Pi_{t}$ is $\Pi_{i}$, and if $\Pi$ is a refinement of each $\Pi_{t}$.

Let $\Pi^{1}, \Pi^{2}, \ldots$ form a mutually independent sequence of partitions of the universal event of a suitable probability space, such that, for each $r, \Pi^{r}$ is an $\left(m_{r}, \varepsilon_{r}\right)$-daisy. Let $\left\{\left(\Pi^{r}\right)_{t}, t \in T\right\}$ be an array of partitions based on $\Pi^{r}$, and let $\delta_{t}$ be the sigma-field generated by the partitions $\left(\Pi^{r}\right)_{t}, r=1,2, \ldots$ Let $A$ be the union of the centers $C^{r}$ of the daisies $\Pi^{r}$.

LEMMA. If $m_{r} \varepsilon_{r} \rightarrow \infty$, then for each $s$

$$
\sup _{t>s} P\left(A \mid \delta_{t}\right)=1 \text { almost surely. }
$$

Proof. As is evident from (1), $m_{r} \varepsilon_{r} \rightarrow \infty$ implies that, for each $s$, $\sup _{t>s} P\left(C^{r} \mid\left(\Pi^{r}\right)_{t}\right) \rightarrow 1$ in distribution as $r \rightarrow \infty$. Thus

Received by the editors December 11, 1978 and, in revised form, February 12, 1979.

AMS (MOS) subject classifications (1970). Primary 60G45.

Key words and phrases. Multi-parameter martingales.

'Research supported by National Science Foundation Grant MCS 77-01665. 
$\sup _{r} \sup _{t>s} P\left(C^{r} \mid\left(\Pi^{r}\right)_{t}\right)=1 \quad$ almost surely.

Since $A$ includes $C^{r}, P\left(A \mid \delta_{t}\right)$ exceeds $P\left(C^{r} \mid \delta_{t}\right)$, which in turn equals $P\left(C^{r} \mid\left(\Pi^{r}\right)_{t}\right)$ because, for each $t$, the partitions $\left(\Pi^{1}\right)_{t},\left(\Pi^{2}\right)_{t}, \ldots$ are independent. Consequently, (2) must hold.

An array $\left\{\Pi_{t}, t \in T\right\}$ is decreasing if $s \leqslant t$ implies $\Pi_{s}$ is a refinement of $\Pi_{t}$. To obtain a decreasing array based on an $(m, \varepsilon)$-daisy $\Pi$, first note that $\Pi_{t}$ is determined for $|t|=m$. Then $\Pi_{t}$ must be the trivial partition for $|t|>m$, and the array can be completed in various ways, for example by setting $\Pi_{t}=\Pi$ for $|t|<m$. Say the decreasing case obtains if for each $r$ the array $\left\{\left(\Pi^{r}\right)_{t}, t \in T\right\}$ introduced above is decreasing. The increasing case is defined analogously.

Plainly, $P\left(A \mid \delta_{t}\right)$ is a uniformly bounded martingale or reversed martingale according as the increasing or decreasing case obtains.

Proposition. Suppose $m_{r} \varepsilon_{r} \rightarrow \infty$ and $\sum \varepsilon_{r}<\infty$. Then, in the increasing case, $P\left(A \mid \mathcal{S}_{t}\right), t \in T$, diverges with positive probability and, in the decreasing case, it diverges with probability one.

Proof. Since the centers $C^{r}$ are independent, $\sum \varepsilon_{r}<\infty$ implies $0<P A<1$. Consider first the decreasing case. For any increasing sequence $t(j) \in T, \cap \delta_{t(j)}$ is part of the trivial tail sigma-field of the independent sequence of partitions $\Pi^{1}$, $\Pi^{2}, \ldots$ because $m_{r} \rightarrow \infty$ and $\left(\Pi^{r}\right)_{t}$ is the trivial partition for $|t|>m_{r}$. Thus $P\left(A \mid \delta_{t(j)}\right)$ converges almost surely to the constant $P A<1$. This, together with (2), implies that $P\left(A \mid \delta_{t}\right)$ diverges almost surely. Consider now the increasing case. If $|t|>m_{r}$, then $C_{r}$, the center of $\Pi^{r}$, is $\left(\Pi^{r}\right)_{t}$-measurable, and hence $\delta_{t}$-measurable. So if $t(j)=(j, j)$, each $C^{r}$ and, hence, $A$, is measurable relative to the limit of the $\delta_{t(j)}$. Therefore, by Levy's martingale convergence theorem, $P\left(A \mid \delta_{t(j)}\right)$ converges to zero almost everywhere off $A$ (and to 1 almost everywhere on $A$ ). This, together with (2), implies that $P\left(A \mid \delta_{t}\right)$ diverges almost everywhere on the complement of $A$.

As is easily verified, a uniformly bounded martingale parameterised by $T$ which diverges almost surely is $M_{t}=\sum M_{t}^{n} / 2^{n}$, where $\left(M_{t}^{1}\right),\left(M_{t}^{2}\right), \ldots$ is a sequence of independent copies of the martingale described above.

Of course, examples such as these indicate the necessity of some auxiliary condition to guarantee the almost sure convergence of multi-parameter martingales. The last word on this subject does not yet seem to have been said, but some such supplementary conditions can be found in the references.

\section{REFERENCES}

1. R. Cairoli, Une inégalité pour martingales à indices multiples et ses applications, Séminaire de Probabilités IV, Lecture Notes in Math., vol. 124, Springer-Verlag, Berlin, 1970, pp. 1-28.

2. R. Cairoli and J. B. Walsh, Stochastic integrals in the plane, Acta Math. 134 (1975), 111-183.

3. J. Dieudonné, Sur un théorème de Jessen, Fund. Math. 37 (1950), 242-248.

4. L. E. Dubins and J. W. Pitman, A pointwise ergodic theorem for the group. of rational rotations, Trans. Amer. Math. Soc. 251 (1979), 299-308. 
5. A. Gut, Convergence of reversed martingales with multidimensional indices, Duke Math. J. 43 (1976), 269-275.

6. K. Krickeberg, Convergence of martingales with a directed index set, Trans. Amer. Math. Soc. 83 (1976), 313-337.

7. J. Neveu, Discrete parameter martingales, North-Holland, Amsterdam; American Elsevier, New York, 1975.

8. R. T. Smythe, Sums of independent random variables on partially ordered sets, Ann. Probability 2 (1974), 906-917.

Department of Mathematics, Universtty of California, Berkeley, California 94720

Department of Statistics, University of California, Berkeley, California 94720 